This work is licensed under a Creative Commons Attribution 4.0 International License.

Ovaj rad dostupan je za upotrebu pod licencom Creative Commons Imenovanje 4.0 međunarodna.

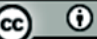

Renata CORNEJO

J. E. Purkyně-Universität in

Ústí nad Labem, Tschechien

Pasteurova 3544/1, 40096 Ústí

nad Labem-město

renata.cornejo@yahoo.de
UDK 821.112.2.09 Faktor, J.-3

821.112.2(436).09 Stavarič, M.-3

DOI: https://doi.org/10.29162/ANAFORA.v8i2.9

Origineller wissenschaftlicher Beitrag

Original Research Article

Erhalten am 1.7. 2021

Received: 1 July 2021

Angenommen am 31.8. 2021

Accepted: 31 August 2021

\title{
KRANKHEITSBILDER IM ROMAN SCHORNSTEIN VON JAN FAKTOR UND TERMINIFERA VON MICHAEL STAVARIČ
}

\section{Zusammenfassung}

Der Beitrag beschäftigt sich mit den Entwürfen von Krankheitsbildern in den Prosawerken Schornstein (2006) von Jan Faktor und Terminifera (2007) von Michael Stavarič und befragt die Texte im Hinblick auf die Exklusionsmechanismen, die sich an der Marginalisierung und zunehmenden ,Pathologisierung' beider Hauptfiguren beteiligen. Im Vordergrund steht dabei die Frage, wie sich die Wahrnehmung der Hauptfiguren durch die Außenwelt auf deren Selbstwahrnehmung und Handeln auswirkt. Nicht zuletzt wird überlegt, inwiefern die hier entworfenen Krankheitsbilder als eine Parabel bzw. ein Abbild der ,Krankheiten' unserer heutigen Welt gelesen werden können.

Schlïsselwörter: Deutsche Literatur, Österreichische Literatur, Jan Faktor, Michael Stavarič, Krankheitsbilder 


\section{Einleitung}

„Wenn ich träume, gehört mir die Welt ganz allein. Im Kopf, da kann einem keiner gefährlich werden." (Stavarič 7) lautet der erste Satz des Prosawerkes Terminifera von Michael Stavarič. Die Welt im Kopf wird somit gleich am Anfang als eine ,eigene' markiert, wobei sich das ,Eigene' bald als eine geistige und psychische Störung entpuppt. Die künstlerische Darstellung von Krankheiten in der Literatur hat eine lange Tradition. Wie Susan Sontag in ihrem Essay Krankheit als Metapher (1977, dt. 1978) dargelegt hat, erfreuen sich bestimmte Krankheiten in Kunst und Literatur besonderer Vorliebe - so z. B. Tuberkulose oder Krebs, die sich besonders als Projektionsflächen eigener Befindlichkeit (vgl. Roman Mars von Fritz Zorn, 1977) oder zu ästhetisierenden Interpretationen (vgl. Roman Der Zauberberg von Thomas Mann, 1924) eignen. Genauso wurde auch genügend auf den engen Zusammenhang zwischen einer psychischen Erkrankung und künstlerischer Kreativität hingewiesen - ein Beispiel der literarischen Bearbeitung dieses Phänomens stellt zweifelsohne der Faustus-Roman (1947) von Thomas Mann dar, in dem er das Leben des Tonsetzers Adrian Leverkühn aus der Perspektive des Freundes Serenus Zeitblom erzählen lässt und am Beispiel des kongenialen Komponisten ein überzeugendes Krankheitsbild der voranschreitenden Syphilis nachzeichnet, nachdem sich Leverkühn bewusst anstecken lässt und so seinen Tribut an den Teufel für seine Genialität zahlt. Bereits in diesem Text fungiert das Krankheitsbild als Metapher für ein ,krankes' System eines ganzen Landes unter Hitlers NS-Herrschaft.

Auf den Diskurs der Wertung und Normvermittlung von ,krank' und ,gesund' in den sozialen und kulturellen Praktiken hat bereits 1989 Thomas Anz in seiner Publikation Gesund oder Krank? Medizin, Moral und Ästhetik in der deutschen Gegenwartsliteratur hingewiesen, in der er an konkreten Beispielen aus der deutschen Literatur vom 18. Jahrhundert bis in die Gegenwart aufgezeigt hat, welche weitreichende normative Kraft beide Begriffe - Krankheit und Gesundheit - in unserer europäischen, logozentrisch und hierarchisch gedachten Kultur besitzen und wie sich ein Gegenmodell zu diesem traditionellen Verständnis von Zivilisation und Rationalisierung in der Moderne etabliert. In den literarischen Texten der Moderne fungiert Krankheit häufig als Metapher für Tod, Eros, Traum, mythische Ekstase u. Ä. Seit der Moderne bezieht sich Krankheit zunehmend nicht mehr auf bloße empirische Erfahrung, sondern steht vielmehr stellvertretend für einen gesellschaftlichen Diskurs. In der Gegenwartsliteratur werden schließlich das Motiv und der literarische Topos 
der Krankheit vor allem als ein Gegenentwurf zum Rationalitätsdiskurs und ein Gegenbild zur leistungsorientierten Konsumgesellschaft eingesetzt, die auf diese Weise kritisiert oder karikiert wird.

Sowohl Michael Stavarič (geb. 1972 in Brno) als auch Jan Faktor (geb. 1951 in Prag), deren Werke im Folgenden im Hinblick auf Krankheitsbilder und deren Funktion im Text näher untersucht werden, wurden in der ehemaligen Tschechoslowakei geboren und repräsentieren die deutsche (Jan Faktor) und österreichische (Michael Stavarič) Gegenwartsliteratur. Der seit 1978 in Ost-Berlin lebende Jan Faktor veröffentlichte 2006 seinen ersten (mit dem Alfred-DöblinPreis ausgezeichneten) Roman Schornstein, der seit 1979 in Österreich ansässige Michael Stavarič publizierte ein Jahr später seinen zweiten Prosatext Terminife$r a$ (2007). Beide Werke entwerfen am Beispiel ihrer Hauptfiguren eindringliche Krankheitsbilder, die ein Abbild der heutigen ,kranken' Welt zeichnen und in diesem Sinne als überzeitliche Parabeln gelesen werden können.

\section{Der Kampf gegen die Krankheit als Kampf gegen das ,marode‘ System}

Der Debütroman Schornstein von Jan Faktor wurde von der Literaturkritik mit Begeisterung als Gegenwartsroman begrüßt, „der auf rätselhafte Weise aus den Themen Krankheit und Depression Heiterkeit und gute Laune hervorzaubert" (Magenau 1). In seiner Laudatio bei der Vergabe des Alfred-Döblin-Preises 2005 verortet Magenau den Roman wegen seiner „lakonischen“Erzählweise in der Tradition eines Schelmenromans (6). Am Anfang des Romans steht das „Ende“, das dem Leser einen Einblick in die aktuelle Situation des Ich-Erzählers vermittelt, bevor der gleichnamige Ich-Erzähler Schornstein, an einer seltenen, lebensgefährlichen Blutkrankheit leidend, im zweiten Kapitel mit der Überschrift „Am Anfang“" seine Krankheitsgeschichte (die sich allmählich in einen Überlebenskampf verwandelt) von Anfang an zu erzählen beginnt:

Ich war lange davor wirklich schon einmal so etwas wie eine halbe Leiche - und potentiell bin ich es immer noch. Mit dreiunddreißig, also vor zehn Jahren, war es schon so weit mit mir, daß ich beim gemütlichen Herumlaufen wie ein Greis stehen bleiben mußte, um nicht umzufallen. Und ich bin dann sogar umgefallen, wobei mir auf dem Boden gar nicht klar war, daß das mein Ende hätte sein können. (Faktor 15)

Schornstein, der gerade einen Herzinfarkt überlebt hat, kann nur durch eine regelmäßige ,Blutwäsche` mit einer Maschine am Leben erhalten werden, die 
sein Blutplasma vom tödlichen Blutfett säubert. Sein Leben ändert sich ruckartig mit einem Anruf seines Hausarztes, der ihm mitteilt, dass seine Krankenkasse die finanziell aufwändige Blutsäuberungstherapie nicht mehr übernehmen kann, was einem Todesurteil gleicht. Gleichzeitig mit dieser lebensbedrohlichen Mitteilung der Kassenärztlichen Vereinigung (weiter „KV“) bricht das Wasser nach einem Gewitter durch die Zimmerdecke in das bis dahin ruhig ablaufende Leben Schornsteins ein - ein Vorzeichen des sich anbahnenden Kampfes um das eigene Überleben. Spätestens im Kapitel „Kohlhaas“ wird klar, dass Schornstein gegen ein durchaus komplexes, gut organisiertes und gesellschaftlich sanktioniertes System zu kämpfen haben wird, welchem gegenüber ein Einzelner kaum eine Chance auf Erfolg hat und zudem der Gefahr unterläuft, von diesem ,Moloch - hier namens Kassenärztliche Vereinigung - erbarmungslos zermalmt zu werden. Ähnlich wie in Kleists Kohlhaas, wo im Mittelpunkt der Streit zwischen den mittelalterlichen und frühabsolutistischen Rechtsvorstellungen steht, muss auch Schornstein das Recht des Einzelnen, die ungesetzlichen Handlungen der ,Obrigkeit' zurückzuweisen, in die eigenen Hände nehmen und versuchen, mit seinem Problem bei den zuständigen Ämtern und Behörden , anzukommen', was sich als höchst problematisch, wenn nicht unmöglich erweist. Denn wie in Kafkas $S c h l o \beta^{1}$ stößt er immer wieder an Instanzen, die Institutionen mit ihren Eigeninteressen vertreten und keine ,Menschen' vor Augen haben, die in ihrer Hierarchie zwar klar strukturiert, jedoch in Zuständigkeitsbereichen vollkommen undurchsichtig und undurchschaubar konzipiert sind:

Von mir aus hätte die KV der verlängerte Arm der Ärztekammer sein können, deren Tochterfirma oder eine Untergrundarmee kassenversklavter Ärzte. Irgendwo vermutete ich allerdings eine Dachorganisation, vor allem auch eine klar strukturierte Hierarchie, auf deren Gipfel ein Minister etwas zu sagen hat. (16)

Zwar gelingt es Schornstein bis zur höchsten Instanz Dr. Dr. Horn, „dem lipidologischen Experten der mächtigen KV-Kommission“ (277) zu gelangen, aber auch dort erhält er keine Antwort auf seine Fragen und muss feststellen, dass er Opfer eines ,Täuschungsmanövers ${ }^{\iota_{2}}$ wurde und sich selbst helfen muss (das gelingt ihm, indem er einen privaten Sponsor findet). Allmählich verfällt Schornstein in Depressionen, er schwankt immer stärker zwischen einer resig-

\footnotetext{
${ }^{1} \mathrm{Zu}$ den intertextuellen Bezügen in Schornstein zu Kafkas Schloß vgl. Cornejo 2010.
} 
nativen und passiven Haltung einerseits und panischen, ihn vollkommen lähmenden Angstzuständen andererseits:

Wenn das, was in mir im Gange war, der beginnende Wahnsinn sein sollte, dann wurde das, was im allgemeinen so genannt wurde, dümmlich glorifiziert. Die Leute wissen wahrscheinlich nicht, wovon sie reden. Dieses langweilige Brüten war furchtbar leise und ereignislos und zum Aufgeilen vollkommen ungeeignet. Der wenig aufregende Satz, Mir geht es nicht gut' ist gar nicht so übel, dachte ich. Eigentlich enthält er schon alles. (174)

Auf Rat seiner Frau sucht er schließlich einen Psychoanalytiker auf. Dr. Brakwart, der sich als Hobby-Historiker mit Vorliebe für das Thema Deutschland und das 20. Jahrhundert entpuppt, gelingt es während der Therapie-Gespräche in Schornstein allmählich durch Bilder und phantasierte Geschichten dessen verschüttete, jedoch emotional wichtige Inhalte zum Vorschein zu bringen - das langjährige Verdrängen der eigenen jüdischen Identität. Als dieser Teil seiner Persönlichkeit durch Erinnerungen an die Kindheit ,freigelegt' und ihm bewusst wird, dass die lustigen Gute-Nacht-Geschichten in Wirklichkeit KZ-Erlebnisse und fehlgeschlagene Selbstmordversuche der weiblichen Familienmitglieder sind, ergibt plötzlich alles einen Sinn: Denn die KV, die ihm die Bezahlung seiner lebensnotwendigen Bluttherapie verweigert, ist eine noch unter dem „Dritten Reich“ in Nazi-Deutschland gegründete und bis heute problemlos weiterhin bestehende Institution, die - in höchst ironischer Umkehrung - jetzt wieder einem ,Juden' nach dem Leben trachtet. Nicht nur die Hauptfigur und die ganze Familie Schornstein sind Opfer ihrer eigenen Verdrängungsstrategien, sondern - wie sich herausstellt - die ganze Gesellschaft: auf institutioneller Ebene vertreten durch die KV, „eine reine Nazigründung“ (257), auf der individuellen Ebene repräsentiert durch Schornsteins Nachbarin Frau Schwan, die die Nazi-Zeit (da ihre Jugendzeit) nicht nur verklärt und glorifiziert, sondern auch die SS-Uniform ihres verstorbenen Mannes im Schrank in tadellosem Zustand aufbewahrt und nahezu rituell verehrt, während sie selbst sowie ihre ganze Wohnung einen unerträglichen Gestank wie ein Stigma um sich verbreiten. An diesem Punkt angelangt, wird der Ich-Erzähler zunehmend ,unzuverlässig', die ,wahren' Geschichten sind nicht mehr von den Phantasiegeschichten zu unterscheiden, einige scheint er dem Psychoanalytiker als bloßes ,Analysematerial' zu liefern, anderes wird nur dem Leser anvertraut. Die Grenzen zwischen Kranksein und Gesundsein werden sowohl für Schornstein und 
seine Frau als auch für den Leser „immer verschwommener“ (Gmehling 120). Schließlich beendet Schornstein die Sitzungen und nimmt sein Leben selbst in die Hand. Der Lauf um sein Leben endet symbolisch (gemeinsam mit seiner Frau Anne, die ihm die ganze Zeit den Rücken stärkt und die wichtigste Stütze ist) auf dem Dach des zu reparierenden Hauses, denn „[h]ier oben ist es nicht schwer, die Dinge positiv zu sehen“" (Faktor 280$)^{3}$ - über die semantisch neu belegten ,Schornsteine“ hinweg. Und so schließt sich der Kreis und das „Ende“, das am Anfang des Romans steht, kann noch einmal gelesen werden, diesmal mit einer neuen Perspektivierung: Die Katastrophe der letzten zehn Monate hat dem Ich-Erzähler zwar zugesetzt, aber die Hoffnung auf Besserung schimmert durch. Der vor dem Supermarkt neben einer offenen Konservenbüchse kotzende Mann in der Eingangsszene des Romans wird zum ebenbürtigen Ebenbild des Ich-Erzählers und nimmt die ganze folgende Handlung samt deren Ausgang vorweg, indem der Kotzende, wie Schornstein auch, trotz seines krankhaften Zustandes die innere Balance beibehalten und aufrecht seinen Weg fortsetzen kann.

\section{Die Ich-Spaltung der Persönlichkeit als Folge der Ausschlussmechanismen der Außenwelt}

Stavaričs Terminifera zeichnet das Bild eines typischen Außenseiters, der sich durch das Verhalten der Außenwelt und die ihm zugefügten Schädigungen zu einem psychisch kranken Menschen entwickelt. Der Ich-Erzähler namens Lois wird in seiner Phantasie von Monstern heimgesucht, die ihn seit seiner Kindheit begleiten, in der Nacht heimsuchen und ihm Angst einjagen. Von der Außenwelt wird er wegen seiner ,Andersartigkeit', die nicht näher beschrieben wird, als eine Art Monster und Ungeziefer betrachtet und häufig schikaniert. Es überrascht deswegen nicht, dass sich der Ich-Erzähler mit einem „Monster, braun im Schritt, mit großen Flügeln“, dessen wissenschaftlicher Name „Chortoicetes Terminifera" lautet, identifiziert - mit einer australischen Wanderheuschrecke also, die „, [r]asch in die Freiheit“ (Stavarič 19) springen kann, wogegen sich Lois wie in einem Gefängnis gefangen fühlt. Lois arbeitet als Krankenpfleger in einem Wiener Krankenhaus, kann jedoch keine engeren Beziehungen

\footnotetext{
${ }^{3}$ Gmehling (2019) untersucht in seiner Studie intensiver konkrete und metaphorische Räume im Roman Schornstein, darunter auch die konkreten Makro- und Mikroräume der erzählten Welt, Räume mit marginalem, ,hybridem ' und heterotopem Charakter, Grenzen und Grenzüberschreitungen, Räume der Macht und Ohnmacht sowie auch den Raum der Sprache. Im Vordergrund stehen weiterhin die narrative Darstellung und Semantik des erzählten Raumes.
} 
zu Menschen in seiner Umgebung entwickeln - die einzige Ausnahme bilden die Krankenschwester Mona, mit der Lois befreundet ist, und sein treuer Hund Sammy. Sehr bald wird deutlich, dass die Gründe für Lois' fehlende soziale und emotionale Kompetenz sowie die psychischen Probleme in dessen Kindheit zu suchen sind, die er als Findling in einem Kinderheim verbringen musste - nicht nur ungeliebt, sondern sowohl von den anderen Kindern als auch von den Heimangestellten tagtäglich verbal malträtiert, psychisch schikaniert und nicht zuletzt auch physisch missbraucht: „Ich bin die Treppe hinuntergefallen, gegen eine Tür gelaufen, im Bad ausgerutscht. Nein es tut nicht weh, Herr Doktor: Passiert bestimmt nicht wieder. [H. i. O.] Der Doktor fasst an die Hoden, ob es da auch weh tut.“ (34) Die im Kinderheim erlebten Misshandlungen und Traumata verwandeln sich in angst- und furchterregende Monster, die ihn Tag und Nacht heimsuchen und vor denen sich Lois in eine Phantasiewelt der Comic-Helden flüchtet. So bewegt er sich ständig an der Grenze zwischen Traum und Wirklichkeit. ${ }^{4}$ Der Vulkanier Mr. Spock, eine Star-Trek-Figur, die halb Vulkanier und halb Mensch ist und deswegen nur sehr abgeschwächt Empfindungen und Gefühle hat, wird zu Lois' Alter Ego und Stütze, die ihm Kraft gibt, das Geschehene ungeschehen zu machen, das Erlebte zu vergessen und die eigene Schwäche in innere Stärke umzuwandeln. Die Grundidee dazu erklärt Stavarič in seiner Salzburger Stefan Zweig Poetikvorlesung wie folgt:

Wenn Superman morgen aufwacht, dann ist er Superman. Sein Alter Ego ist Clark Kent. [...] Was er als Clark Kent trägt - die Brille, den Businessanzug - das ist das Kostüm [...], das Superman trägt, um sich uns anzugleichen. Clark Kent ist das, wie Superman uns sieht. Und was sind Clark Kents Charakteristika? Er ist schwach, unsicher, er ist ein Feigling. Clark Kent ist Supermans Kritik an der ganzen Menschheit. (Stavarič 111-12)

In der Erfindung von Mr. Spock, zu dem sich Lois in seiner Hilflosigkeit als Phantasiefigur flüchtet und dessen Stärke und Unempfindlichkeit er als Schutzmechanismus annehmen zu können glaubt, zeichnet sich die bereits beginnende Ich-Spaltung des Ich-Erzählers, die sich auf ironische Weise auch in seinem Spitznamen „Lois“ widerspiegelt (der Ich-Erzähler bleibt namenlos). Diesen bekommt er in Anlehnung an Supermans Freundin: „Sie sagten, wenn du die passende Statur hättest, du wärst wie Superman [H. i. O.]. Wenn ich nur nicht

\footnotetext{
${ }^{4}$ Näher zu den Grenzüberschreitungen im Werk von Michael Stavarič vgl. die Studie Im Niemandsland zwischen den Grenzen (Bartl 2018).
} 
so dünn wäre. Wir nennen dich Lois. Lois Lane [H. i. O.]. Supermans Freundin.“ (Stavarič 68-69) Wie weit die psychischen Schädigungen und Deformierungen gehen und inwiefern sie das gegenwärtige Leben von Lois prägen und beeinflussen, wird im Text nur sporadisch angedeutet. So z. B. wenn die Ärztin Kristina, die zu Lois gern eine engere Beziehung aufbauen würde, aber dabei nur auf distanzierte Gefühlskälte und ein „Herz aus Stein“ (87) stößt, andeutet, dass sie um dessen Geheimnis (wohl sexueller Natur) Bescheid weiß: „Kristina hat zu mir gesagt, sie wüsste alles, alles von dir und Sammy! [H. i. O.]“ (87) Dass Lois' Beziehung zu Sammy eine ,besondere` ist, darauf wird im Text mehrmals an unterschiedlichen Stellen hingewiesen - nicht zuletzt auch am Ende des Romans, als der Hund, der das Haus bewacht, von einem Dieb brutal getötet wird und Lois dessen Tod mit den folgenden Worten kommentiert: „Sammy war mir eine gute Frau" (146). Die voranschreitende psychische Störung auf Grund der von Menschen in der Kindheit erfahrenen Misshandlungen führt bei Lois im Erwachsenenalter zur Unmöglichkeit, emotionelle Bindungen zu Menschen zu entwickeln. Gefühle und Liebe werden statt auf Menschen auf Tiere übertragen, in diesem Fall auf einen Hund: „Da tötet also einer den letzten aufrichtigen Menschen auf dieser Welt wegen eines tragbaren Fernsehers mit Zimmerantenne.“ (146) Der Hund wird als ehrlicher und aufrichtiger, also „menschlicher“ als ein Mensch empfunden und geschätzt, denn „[w]enn man einem Hund erst einmal in die Augen schaut, dann weiß man, der lügt nie. Da passiert nichts ohne einen triftigen Grund.“ (38)

Die Ich-Spaltung der Persönlichkeit, die ihren Ausdruck in der Projektion einer Alter-Ego-Beschützerfigur aus der Star-Trek-Welt findet, spiegelt sich auch in der Verunsicherung der Geschlechteridentität wider. ${ }^{5}$ Während der Doktor (Kinderarzt) bei Lois nach den Misshandlungen im Kinderheim statt entschieden dagegen einzuschreiten, seine Hoden betatscht, spricht Lois von sich an mehreren Stellen als von einem Mädchen (von seiner ersten Periode oder gar Entjungferung). Ähnlich wie Andri in Max Frischs Andorra verinnerlicht Lois das Bild, das sich die anderen wegen seiner schmächtigen Figur von ihm machen und lässt sich zum Objekt der Begierde sexualisieren: „Du bist

\footnotetext{
${ }^{5}$ Zur Androgynie der Hauptfigur in Terminifera vgl. Bartl 2010. Bartl interpretiert die Figur Lois als androgyne Bastardfigur, die zunächst als „das Kind“ jenseits geschlechtlicher Fixierung steht, doch nach der Einweisung ins Kinderheim anderen Mechanismen der Sozialisation unterworfen wird und männliche wie weibliche Identitäten annimmt.
} 
ein kluges Mädchen, das sieht man schon von Weitem.“ (28) oder „Mädchen, du musst fortan ganz auf deine Titten setzen. [H. i. O.]“(53)

Der Ausgang der Geschichte ist unsicher, wie auch die Erzählweise und die fragile Identität der Hauptfigur. Nachdem Mr. Spock als die stärkere Hälfte des Ich verabschiedet wird und verschwindet, ${ }^{6}$ verfällt die Hauptfigur in Apathie und Gleichgültigkeit gegenüber seiner Umgebung. Nachdem nun der einzige Freund, die Hündin Sammy tot ist, scheint es nicht viele Möglichkeiten für den Ich-Erzähler zu geben. Ohne es auszusprechen, bleibt nach der Zerstörung der einzigen emotionalen Bindung der Hauptfigur zu einem Lebewesen auf dieser Welt eigentlich nur eins: die Absage an eine solche Welt und der Gedanke an das freiwillige Scheiden daraus.

\section{Fazit}

Abschließend und zusammenfassend kann gesagt werden, dass beide Werke Krankheitsbilder entwerfen, anhand deren die heutige Welt kritisiert (Stavarič) oder auch karikiert (Faktor) wird. Schornstein leidet an einer körperlichen (physischen) Krankheit, deren Behandlung für das Krankensystem zu kostenaufwendig ist und deswegen, d. h. aus rein pragmatischen/finanziellen Gründen, darauf verzichtet werden bzw. ein Menschenleben geopfert werden soll. Der krankhafte Prozess im Körper der Hauptfigur wird schrittweise in Bezug zu einem ,maroden' Krankenkassensystem gesetzt, das auf die ganze, stark konsum- und leistungsorientierte sowie den eigentlichen menschlichen Bedürfnissen entfremdete Gesellschaft übertragen werden kann. Eine weitere, tiefere Bedeutungsebene erhält der Begriff, Krankheit` im Laufe der Handlung, als sich herausstellt, dass die KV eine Gründung noch aus der Nazi-Zeit ist und dass Schornstein eigentlich ein Jude ist. Damit wird direkt die massenhafte Verfolgung und Vernichtung der Juden im Dritten Reich geschichtlich abgerufen und Schornsteins Kampf ums Überleben gewinnt somit unerwartet an historischer Dimension. Beide - sowohl er als auch die Gesellschaft, in der er lebt - bedienen sich, was ihre Vergangenheit angeht, bestimmter Verdrängungsmechanismen, die, wenn sie von Dauer sind, zu einem krankhaften, neurotischen Zustand, einem „einzige[n] Dauerkranksein“ (Faktor 263) führen können, zu einem Zustand, der metaphorisch vom Einzelnen auf die ganze Gesellschaft übertragen werden kann.

\footnotetext{
${ }^{6}$ Zum Thema Grenzfigur, Hybridität und Differenz in Terminifera vgl. Cornejo 2010.
} 
In Stavaričs Werk wird das Krankheitsbild einer Ich-Spaltung der Persönlichkeit als Folge der Misshandlungen in der Kindheit entworfen. Es wird also eine psychische Erkrankung in den Mittelpunkt gestellt, die sich auf Grund des ,krankhaften' Verhaltens der Umgebung gegenüber dem ausgesetzten und im Kinderheim aufgezogenen Kind entwickelt. Als kleines, wehrloses Kind muss die Hauptfigur verbale und körperliche Gewalt in Form von Schikane und auch sexuellem Missbrauch über sich ergehen lassen, welche es nicht in der Lage ist zu bewältigen. Die Folge ist - als Schutzmechanismus - einerseits die innere Spaltung der Hauptfigur und die Erfindung eines stärkeren Ich, das sich zur Wehr zu setzen weiß (in der Kindheit) und andererseits die totale emotionale Unfähigkeit, eine gefühlsmäßige Bindung zu einem anderen Menschen einzugehen (im Erwachsenenalter). Die Bindung gelingt nur zu einem Tier. Bei Stavarič fungiert also das Krankheitsbild als Abbild einer menschenfeindlichen, gewalttätigen und brutalen Gesellschaft, in der der Schwächere untergeht und nur der Starke überleben kann. Hierbei handelt es sich um eine offene Gesellschaftskritik, denn es sind die Erzieher und der Arzt, d. h. Instanzen, die ein Kind zu beschützen haben, die hier vollkommen versagen und nicht nur das Vertrauen des Kindes missbrauchen, sondern selbst tätlich werden.

So gesehen können beide Werke als Parabeln bzw. Abbild der ,Krankheiten unserer heutigen Zeit gelesen werden - das erstere tut es in leicht ironischer Art und Weise und in höchst lakonischer Sprache (Faktor), das zweite offenbart das Ausmaß der Gewaltbereitschaft und verdeckten Brutalität in unserer Gesellschaft anhand von subtilen Andeutungen und in einer höchst poetischen Sprache (Stavarič). Beide lüften dann angedeutete Geheimnisse und bringen das Verdrängte und Verschwiegene zur Sprache - mit ganz unterschiedlichem Ergebnis: Während Lois kaum eine Chance auf ein ,normales' Leben bzw. eine Überwindung der traumatischen Erlebnisse in Aussicht gestellt wird (im Gegenteil - die in der Kindheit erlebte Gewalt wiederholt sich in gewisser Weise durch den gewaltsamen Tod des Hundes noch einmal), kann Schornstein die notwendige Distanz gewinnen und von "oben“ (vom Dach) symbolisch einen hoffnungsvollen Blick über die Schornsteine hinweg in die Ferne richten. 


\section{Literaturverzeichnis}

Anz, Thomas. Gesund oder Krank? Medizin, Moral und Ästhetik in der deutschen Gegenwartsliteratur. Metzler, 1989.

Bartl, Andrea. „Im Niemandsland zwischen den Grenzen. Eine Einführung in das Werk Michael Stavaričs. “ Ästhetische Grenzüberschreitungen: eine literaturwissenschaftliche und literaturdidaktische Erschließung des Werks von Michael Stavarič, Andrea Bartl und Ina Brendel-Perpina (Hg.), Königshausen \& Neumann, 2018, S. 43-56.

---. „Schädigung - Bereicherung? Androgyne Bastardfiguren in der deutschsprachigen Migrationsliteratur am Beispiel von Michael Stavaričs ,Terminifera' und Libuše Moníkovás ,Eine Schädigung.“" Bastard. Figurationen des Hybriden zwischen Ausgrenzung und Entgrenzung, Andrea Bartl und Stephanie Catani (Hg.), Königshausen \& Neumann, 2010, S. 207-20.

Cornejo, Renata. „Der ,zähe Landsmann` Franz Kafka im Werk von Libuše Moníková und Jan Faktor." Franz Kafka und Robert Walser im Dialog, Vesna Kondrič Horvat (Hg.), Weidler Buchverlag, 2010, S. 381-405.

---. „Hybridität und Differenz: Grenzfiguren in den Romanen ,Terminifera und ,stillborn von Michael Stavarič. “ Heimat im Wort. Zum Sprachwechsel der deutsch schreibenden tschechischen Autorinnen und Autoren nach 1968. Eine Bestandsaufnahme, Renata Cornejo (Hg.), Praesens Verlag, 2010, S. 282-90.

Faktor, Jan. Schornstein. Kiepenheuer \& Witsch, 2006.

Gmehling, Karl-Heinz. „Raumkonstellationen in Jan Faktors Roman ,Schornstein.“ Brünner Beiträge zur Germanistik und Nordistik, H. 33, Nr. 1, 2019, S. 113-28.

Magenau, Jörg. „In überlebensnotwendiger Distanz. Jan Faktor und sein Roman ,Schornstein.“ Wespennest, Nr. 142, 2006, S. 75-81. https://www.literaturreport.de Zugriff 30. 6. 2021.

Sontag, Susan. Krankheit als Metapher. Hanser, 1978.

Stavarič, Michael. Der Autor als Sprachwanderer. Salzburger Stefan Zweig Poetikvorlesung, Bd. 4. Sonderzahl, 2016. 


\title{
PORTRAYAL OF ILLNESS IN JAN FAKTOR'S SCHORNSTEIN AND MICHAEL STAVARIČ'S \\ TERMINIFERA
}

\begin{abstract}
Renata CORNEJO

J. E. Purkyně-Universität in Ústí nad Labem, Czech Republic

Pasteurova 3544/1, 40096 Ústí nad Labem-město

renata.cornejo@yahoo.de
\end{abstract}

The article deals with the concept of clinical pictures in the prose works Schornstein (2006) by Jan Faktor and Terminifera (2007) by Michael Stavarič. The texts are analysed with regard to the mechanisms of exclusion that contribute to the marginalization and increasing "pathologization" of both main characters. The focus is on the question of how the perception of the main characters by the outside world affects their self-perception and actions. Last but not least, consideration is given to the extent to which the clinical pictures outlined here can be read as a parable or an image of the "illnesses" of our world today.

Keywords: German Literature, Austrian Literature, Jan Faktor, Michael Stavarič, clinical pictures 


\title{
PRIKAZI BOLESTI U ROMANU SCHORNSTEIN \\ JANA FAKTORA I TERMINIFERA \\ MICHAELA STAVARIČA
}

\begin{abstract}
Sažetak
Renata CORNEJO

J. E. Purkyně-Universität in Ústí nad Labem, Češka

Pasteurova 3544/1, 40096 Ústí nad Labem-město

renata.cornejo@yahoo.de
\end{abstract}

Članak razmatra prikaz kliničkih slika u proznim djelima Schornstein (2006.) Jana Faktora i Terminifera (2007.) Michaela Stavariča. Tekstovi se analiziraju s obzirom na mehanizme isključivanja koji doprinose marginalizaciji i rastućoj „patologizaciji“ obojice protagonista. U središte se stavlja pitanje kako doživljaj vanjskog svijeta o glavnim likovima utječe na njihov doživljaj samih sebe i vlastite postupke. Naposljetku se proučava opseg u kojem se dane kliničke slike mogu iščitati kao parabola ili slika „bolesti“ današnjega svijeta.

Ključne riječi: njemačka književnost, austrijska književnost, Jan Faktor, Michael Stavarič, kliničke slike 